Methods The cases were 101 obese (BMI over $30 \mathrm{~kg} / \mathrm{m}^{2}$ ) and controls were 101 non-obese (BMI 18,5-24,9 $\mathrm{kg} / \mathrm{m}^{2}$ ) subjects matched by age and sex over the age of 50 years. There was not significant difference between cases and controls regarding the other main risk factors for osteoporosis (time spent from menopause, diabetes, hyperthyreoidism, alcohol and coffee intake, smoking, drugs causing osteoporosis). All probands in the casecontrol study were examined by radiographic investigation, vertebral morphometry, densitometry and by interviewer administered questionnaire. Radiographs were taken according to standardised protocol and hyperostosis was classified using Resnick criteria. Vertebral deformity was diagnosed with the McClosky-Kanis algorithm. Bone mineral density was measured by DEXA.

Results In the case-control study obesity was significantly associated with hyperostosis of the spine $(p ? 0,05)$ with higher mean values of bone mineral density of the femoral neck (p?0,000), trochanter ( $p$ ?0,000), and lumbar spine p?0,014).

Conclusion According to their result obesity was associated with hyperostosis of the spine and with higher bone mineral density but not with fractures and osteoporosis in the past history.

\section{AB0176 INFLUENCE OF NUTRITION ON BONE MINERAL DENSITY IN PATIENTS WITH ANKYLOSING SPONDYLITIS}

H Franck, T Meurer. Center of Rheumatology, Oberammergau, Oberammergau, Germany

\subsection{6/annrheumdis-2001.615}

Background A sufficient alimentation of vitamin D and calcium is essential in the prevention and treatment of osteoporosis. Patients with ankylosing spondylitis have osteoporosis to a various degree.

Objectives The aim of our study was to look at the influence of nutrition on bone mineral density in our patients with ankylosing spondylitis.

Methods We examined 180 patients with ankylosing spondylitis fulfilled the modified New York criteria. The bone mineral density was measured with DEXA (QDR 4500) at the lumbar spine (LS) and right hip.

Factors of nutrition were examined by a standardised questionnaire.

Results Positive correlations with bone mineral density were only found with wine consumption. However, this correlation was week but was confirmed for both hip and LS bone mineral density measurements.

\begin{tabular}{llll}
\multicolumn{2}{l}{ Abstract AB0176 Table 1} & & \\
\hline Milk & $\mathbf{N}=$ & $\mathrm{r}=$ & $\mathbf{p}=$ \\
Sausage & 180 & 0,082 & 0,277 \\
Coffee & 180 & 0,051 & 0,051 \\
Cheese & 180 & 0,038 & 0,614 \\
Wine & 180 & $-0,019$ & 0,795 \\
& 180 & 0,217 & 0,003 \\
\hline Correlation BMD-hip total with consumption of & &
\end{tabular}

Conclusion No significant correlations between milk and cheese intake with bone mineral density were found in patients with ankylosing spondylitis. Dietetic factors do not seem to influence much bone mineral density in patients with ankylosing spondylitis. Only wine consumption was found to have a influence on bone mineral density as is correlated with bone mineral density in our patients with ankylosing spondylitis.

\section{AB0177 SOME DETERMINANTS OF BONE MASS AMD BONE GEOMETRY IN WOMEN OVER 50}

O Lesnyak, Y Lesnyak, P Sarapultsev. Department of Internal Diseases, Regional Hospital N 1, Yekaterinburg, Russia

10.1136/annrheumdis-2001.616

Background The exact influence of different risk factors of osteoporosis on bone need to be assessed.

Objectives The aim of this study was to assess the influence of some anthropometric and lifestyle factors on bone parameters of forearm in women over 50 .

Methods This was a cross-sectional study included 117 women of 50-79 y.o. Volumetric BMD (trabecular, tBMD, and cortical, cBMD) cortical bone area (CBA) and strength sustained indices (SSI) of radius were measured using pQCT (Stratec-XCT960). The relationship among several factors influencing the bone parameters was assessed using a multiple regression analysis.

Results The age was the independent determinant of cBMD and CBA. BMI positively influenced tBMD. SSI depended on age and height. The years since menopause were not significant after controlling for other covariables. Dietary intake of calcium and smoking contributed a little to variation in bone parameters. Strong predictor of tBMD was professional physical activity in youth (negative correlation), and cBMD, CBA and SSI were influenced positively by current professional physical activity.

Conclusion Thus, different mechanisms might be involved in compact and trabecular bone loss and geometric parameters.

\section{AB0178 ON THE DIAGNOSTICS OF POSSIBLY INHERITED OSTEOPOROSIS}

1'É Lányi, ${ }^{2}$ B Gömör, ${ }^{3}$ I Ratkó. 'I. Department of Rheumatology, Polyclinic of the Hospitaller Brothers of St. John of God, Budapest, Hungary; ${ }^{2}$ Chair of Rheumatology, Faculty of Health Sciences of the Semmelweis University, Budapest, Hungary; ${ }^{3}$ Department of Mathematics, Dennis Gábor College, Budapest, Hungary

\subsection{6/annrheumdis-2001.617}

Background Osteoporosis (OP) is a disease of high occurrence in the older-aged population. Early diagnosis may trigger an efficient medication, which could slow down or even stop the decrease of bone mineral density (BMD), leading so to a noticeably decrease of the risk for bone fracture in old age. Reducing the incidence of osteoporosis is of utmost importance for both the individuals and the society, so it is not only of medical importance to identify individuals with a higher-than-average risk of developing osteoporosis.

Objectives The aim of this work is to investigate the role of inheritance in osteoporosis as mirrored by basic diagnostic methods. It is also of practical interest to establish the applicability of the by now routinely applied dual energy X-ray absorption (DEXA) method for early diagnostics of BMD for patient groups with a suspected tendency to inherited osteoporosis.

Methods The patient study group consisted of 28 motherdaughter pairs. The mothers (age group: 60-85 y) were suffering in a severe form of osteoporosis as shown for each of them by crush fractures of the vertebrae. The study of the BMD values was directed to the group of daughters of mothers suffering from osteoporosis (DMO). For the daughters (29-58 y, average 